\title{
Management of leak after sleeve gastrectomy: outcomes of 73 cases, treatment algorithm and predictors of resolution.
}

\author{
Moataz Bashah ${ }^{1,2} \cdot$ Nesreen Khidir $^{1}(\mathbb{D}) \cdot$ Moamena EL-Matbouly $^{3}$
}

Published online: 9 November 2019

(C) The Author(s) 2019

\begin{abstract}
Introduction Gastric leak post laparoscopic sleeve gastrectomy (LSG) is a severe complication that has been reported in 1.5-3\% of cases. Management algorithms of leak exist; however, no known factors predict the time to resolution. This study aims to share outcomes of our management algorithm of post LSG leak, including the rate of resolution, complications, admission to the intensive care unit, conversion to other techniques, and mortality. To determine if any factors can predict the resolution time.

Methods A retrospective analysis of patients who had LSG leaks and was managed in the main tertiary center in Qatar (January 2012-December 2017).

Results A total of seventy-three patients had post LSG leaks. Fifty-six (76.7\%) underwent LSG outside our center. Thirteen leaks $(17.8 \%)$ were after revisional LSG. Laparoscopic exploration was performed in twenty patients (27.4\%) and feeding jejunostomy in nine patients (12.3\%). Patients were followed up for 12 months. All healed within $8.8 \pm 0.72$ weeks. The resolution rate was $(97.1 \%)$ without surgical conversion, while two patients required fistulo-jejunostomy. No patient died. Complications occurred; re-leak (14.9\%) and splenic abscess (2.9\%). Patients on jejunal feeding had shorter resolution time (HR $=2.7, P=0.018)$, while patients on total parenteral nutrition and post-endoscopic dilatation had $66 \%$ and $50 \%$ increases in the resolution time; ( $\mathrm{HR}=0.34, P=0.026)$ and $(\mathrm{HR}=0.5, P=0.047)$, respectively.

Conclusion Management of post-LSG leak is multimodal. Our clinical experience demonstrated less urge to perform extensive surgical interventions. Patients on enteral feeding had shorter resolution time while patients with sleeve stricture had a longer time to resolution.
\end{abstract}

Keywords Management of leak post sleeve gastrectomy $\cdot$ Predictors of resolution time $\cdot$ Post LSG leak complications

\section{Introduction}

Sleeve gastrectomy is the most commonly performed procedure worldwide [1]. Staple line leakage is the second most common cause of death after bariatric surgery with an overall mortality rate of $0.4 \%$ [2]. Gastric leak after laparoscopic

Nesreen Khidir

Dr_sora4@hotmail.com

1 Department of Bariatric and Metabolic Surgery, Hamad General Hospital, P.O. Box 3050 Doha Qatar

2 Weill Cornell Medical College, Doha Qatar

3 Department of General Surgery, Hamad General Hospital, Doha Qatar sleeve gastrectomy (LSG) is a devastating complication that occurs in 1.5 to $3 \%$ post LSG $[3,4]$. This has significant physical and psychological impacts on patients and their families. Fortunately, the worldwide incidence of leak is decreasing $[5,6]$.

The literature described management algorithms for post LSG leak, as determined by the timing of presentation and clinical and nutritional statuses [4, 7]. However, these algorithms were based on a limited number of patients, and no clear guidance is currently available. Some authors also tried to identify if any factors could cause a leak or affect the management $[5,8]$; however, no known factors have been studied to predict the outcomes of a leak or the time to resolution. This study presents the outcomes of the management algorithm for LSG leaks in patients who were managed in our center (2012-2017). The secondary objective was to determine if any factors can predict the resolution time. 


\section{Objectives}

Primary: to share the outcomes of the management algorithm of LSG leak in the main tertiary center in Qatar, including the rates of resolution, complications, admission to the intensive care unit, conversion to other techniques and mortality.

Secondary: to determine whether some factors can predict the resolution time; patients' gender, BMI, time of presentation, primary versus revisional sleeve gastrectomy, the presence of distal sleeve stricture, the number of the fistulous openings in endoscopy, and type of feeding.

\section{Methods}

A retrospective review of prospectively collected data of patients with post LSG leak and managed in our center between January 2012 and December 2017. The study was approved by the medical research center/Hamad Medical Corporation, "IRB approval number 16208/16."

All post sleeve gastrectomy patients who presented with symptoms and signs of a leak had an abdominal computed tomography (CT scan). A leak was diagnosed by radiological findings of oral contrast extravasation in CT scan or fluoroscopy or by endoscopic findings of fistulous opening. Management algorithm is depicted in Fig. 1. Endoscopy and intervention were performed routinely in all leak patients. Type of intervention was dependent on the number and size of the fistulous openings. Patients with one fistulous opening $<5 \mathrm{~mm}$ had either OVESCO "over the scope" clipping or internal endoscopic drainage with pigtail catheter insertion (Surgeon's preference). Patients with one fistulous opening $>5 \mathrm{~mm}$ had endoscopic stenting. Patients with multiple fistulous openings had endoscopic stenting. In cases of sleeve stricture or twist, combined endoscopic balloon dilatation plus stent placement was performed. The operating surgeon or gastroenterologist performed endoscopic gastric stenting under fluoroscopic guidance. Stents were followed radiologically (fluoroscopy) and endoscopically every 2 weeks or as indicated. Bariatric physicians assessed all patients. Vitamins, proteins, and minerals were closely monitored and replaced. A bariatric/chest physiotherapist followed all patients and was on "both chemical and mechanical" deep venous prophylaxis.

Outcomes According to Primary Objective Resolution rate based on gastrografin fluoroscopy image that showed no contrast extravasation, complication rate (re-leak, pulmonary embolism, deep venous thrombosis, gastrocutaneous fistula, and splenic abscess), the rate of admission to the intensive care unit (ICU), conversion to other techniques, and mortality rate.

Outcomes According to Secondary Objective Predictors of earlier resolution; patients' gender, BMI, time to presentation, primary versus revisional sleeve gastrectomy, presence of distal sleeve stricture or twist on fluoroscopy, the number of the

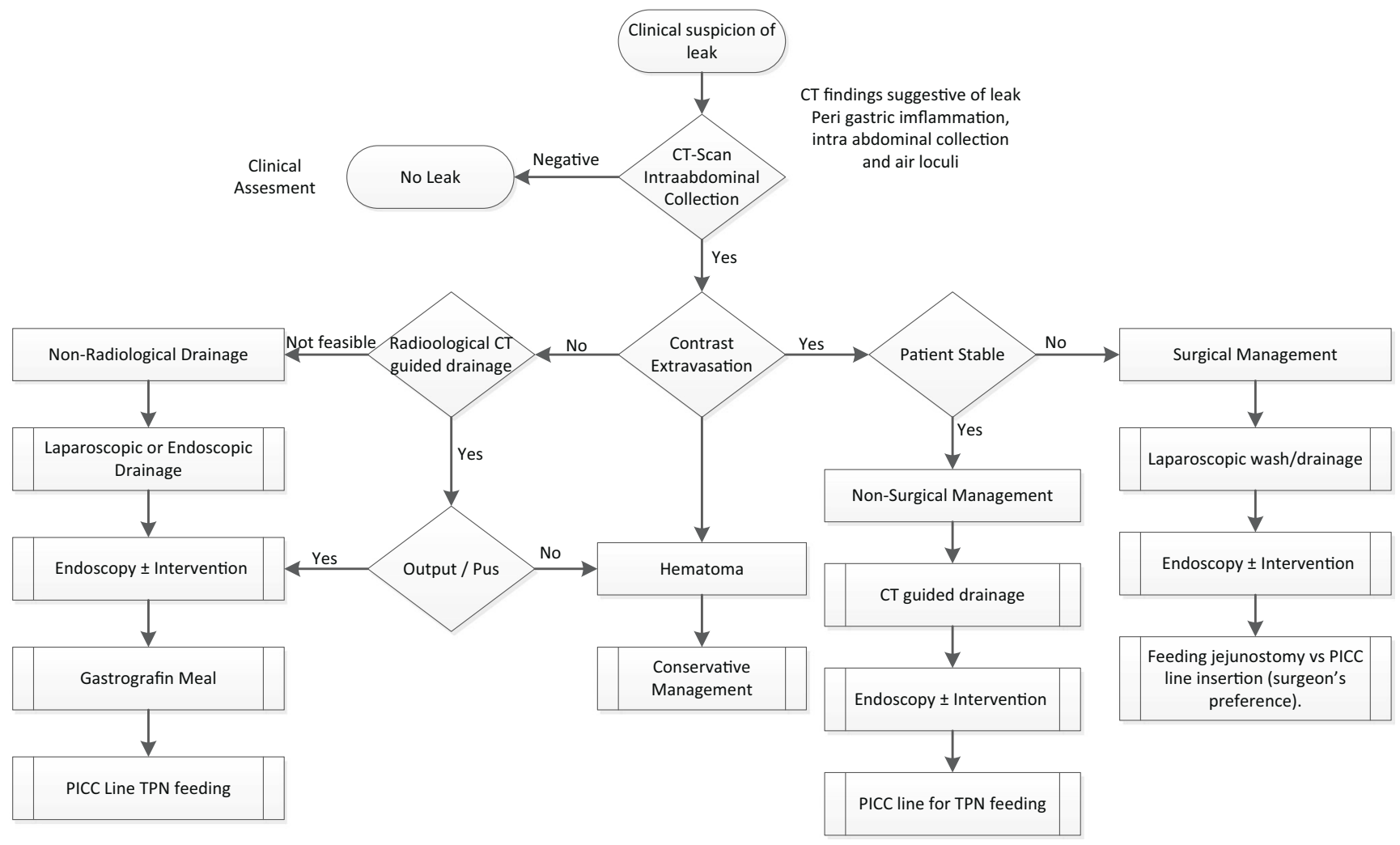

Fig. 1 Management algorithm of patients with post sleeve gastrectomy leak. PICC peripherally inserted central line; TPN total parenteral nutrition 
fistulous openings in endoscopy, and the type of feeding (enteral vs parenteral).

\section{Statistical Analysis}

It was performed using STATA 15 software. Descriptive statistics were provided as frequencies and proportions or means and standard deviations, as appropriate. Statistical significance was set at $P<0.05$. No sample size calculation was done; the study is a retrospective review. Survival analysis was performed using Cox proportional hazard regression test, to investigate the impact of some factors on time to leak resolution. The measure of effect was reported using hazard ratios (HR), 95\% confidence interval $(95 \% \mathrm{CI})$, and $P$ values.

\section{Results}

Out of 4250 patients who underwent LSG in our center, 17 $(0.4 \%)$ leaked. Fourteen $(0.4 \%)$ were primary $(14 / 3748)$, and three $(0.6 \%)$ were revisional $(3 / 502)$. A total of seventy-three leaks were diagnosed and managed. Their demographic details are depicted in Table 1. Fifty-six patients $(76.7 \%)$ had their LSG outside our center. Patients were followed up for a minimum of 12 months and the follow up rate was $93.2 \%$. The BMI at the time of presentation was $42 \pm 8.8 \mathrm{~kg} / \mathrm{m}^{2}$ (95\% CI 40-44). Three patients developed leak during the same admission for surgery, while 70 patients $(95.9 \%)$ were diagnosed after discharge. The patients presented during $19 \pm 16$ days post surgery (95\% CI 15.3-22.8). Sixty patients $(82.2 \%)$ leaked after primary LSG, while thirteen patients $(17.8 \%)$ leaked after revisional LSG. Eleven $(84.6 \%)$ of the revisional cases were after band removal and two cases were post re-sleeve.

Laparoscopic washout drainage was performed in twenty patients $(27.4 \%)$ and feeding jejunostomy was employed in nine

Table 1 Demographic data of patients with post laparoscopic sleeve gastrectomy leak

\begin{tabular}{ll}
\hline Variable & Value \\
\hline Age (years, $M \pm \mathrm{SD})$ & $36.3 \pm 10.6$ \\
Weight $(\mathrm{kg})$ & $117.5 \pm 28.5$ \\
BMI $\left(\mathrm{kg} / \mathrm{m}^{2}, \mathrm{M} \pm \mathrm{SD}\right)$ & $42 \pm 8.8$ \\
Female $(n, \%)$ & $41(56.2 \%)$ \\
Post op day of presentation & $19 \pm 16.1$ \\
ER visits (times, $\mathrm{M} \pm \mathrm{SD})$ & $0.96 \pm 0.75$ \\
DM $(n, \%)$ & $9(12.3 \%)$ \\
$\mathrm{HTN}(n, \%)$ & $13(17.8 \%)$ \\
DM and $\mathrm{HTN}(n, \%)$ & $6(8.2 \%)$ \\
\hline
\end{tabular}

$M$, mean; $S D$, standard deviation; $B M I$, body mass index; $n$, number; $E R$ visits, number of pre-diagnosis visits to the emergency room; $D M$, diabetes mellitus; HTN, hypertension patients $(12.3 \%)$. Sleeve stricture was observed in 12 patients (16.4\%) who underwent balloon dilatation combined with endo-gastric stent placement. Fifty-four patients had endogastric stenting. The management plans are detailed in Fig. 2.

Leak resolution rate was $97.1 \%$ within $8.8 \pm 0.72$ weeks without surgical conversion of fistula (95\% CI 7.4-10.2) while two patients required fistulo-jejunostomy (see Table 2). No mortality occurred. Twenty-two patients were admitted to the intensive care unit (ICU) due to sepsis or septic shock.

To meet the secondary objective of the study, analysis of the patients' gender, BMI, and number of postoperative days to presentation did not impact the time to leak resolution. However, patients who were on jejunal feeding had a substantially shorter resolution time $(\mathrm{HR}=2.7$, CI $1.18-6.15, P=$ 0.018 ), while the patients who were on TPN had $66 \%$ longer time to leak resolution ( $\mathrm{HR}=0.34, P=0.026$ ). Patients who underwent endoscopic dilatation had a $50 \%$ reduction in the resolution time $(\mathrm{HR}=0.5, P=0.047)($ see Table 3$)$.

\section{Discussion}

The gastrointestinal leak is a severe complication of bariatric surgery. Authors reported a decreasing worldwide incidence of post LSG leak to $0.5 \%$ [8]. In our practice, post LSG leak occurred in $0.4 \%$ of cases. To our knowledge, this manuscript reports the most significant number of LSG leaks that were treated in a single center. Our patients were managed according to their clinical status and radiological and endoscopic findings. The treatment options considered the classification of the leak into early, intermediate, and late. All patients post LSG with suspicious clinical picture had CT-abdomen. Only 97.1\% of CTs showed an intra-abdominal collection (Fig. 2). Unfortunately, data of patients with false negative findings of the collection was not available. The surgeon's clinical judgment is crucial; patients who do not look well post-bariatric surgery should raise the suspicion index of a leak. Unstable patients mandated quick surgical wash and drainage.

Our clinical experience presumes that there is less urge to perform reoperation or laparotomies. Approximately $97.1 \%$ of our patients healed their leak without surgical conversion. Comparing our results to others who shared their treatment algorithm, Nimeri et al. reported that $21 \%$ of patients required conversion to an extensive laparoscopic procedure [7]. By contrast, in our group, none required extensive laparoscopic intervention nor laparotomies. Also, a zero-mortality rate was compared with an international leak-related mortality of $0.11 \%$ [9]. No surgical trials were performed to close the fistula. The aim was to avoid tearing the inflamed friable tissues. In agreement with others on that most patients who underwent suturing for their fistula and the fistula failed to close, due to persistence of the leak or failure of the suture [10]. 
Fig. 2 Management details of post sleeve leak patients according to our management algorithm. All results based on 73 patients; OVESCO over the scope clipping; combined: both stenting and OVESCO

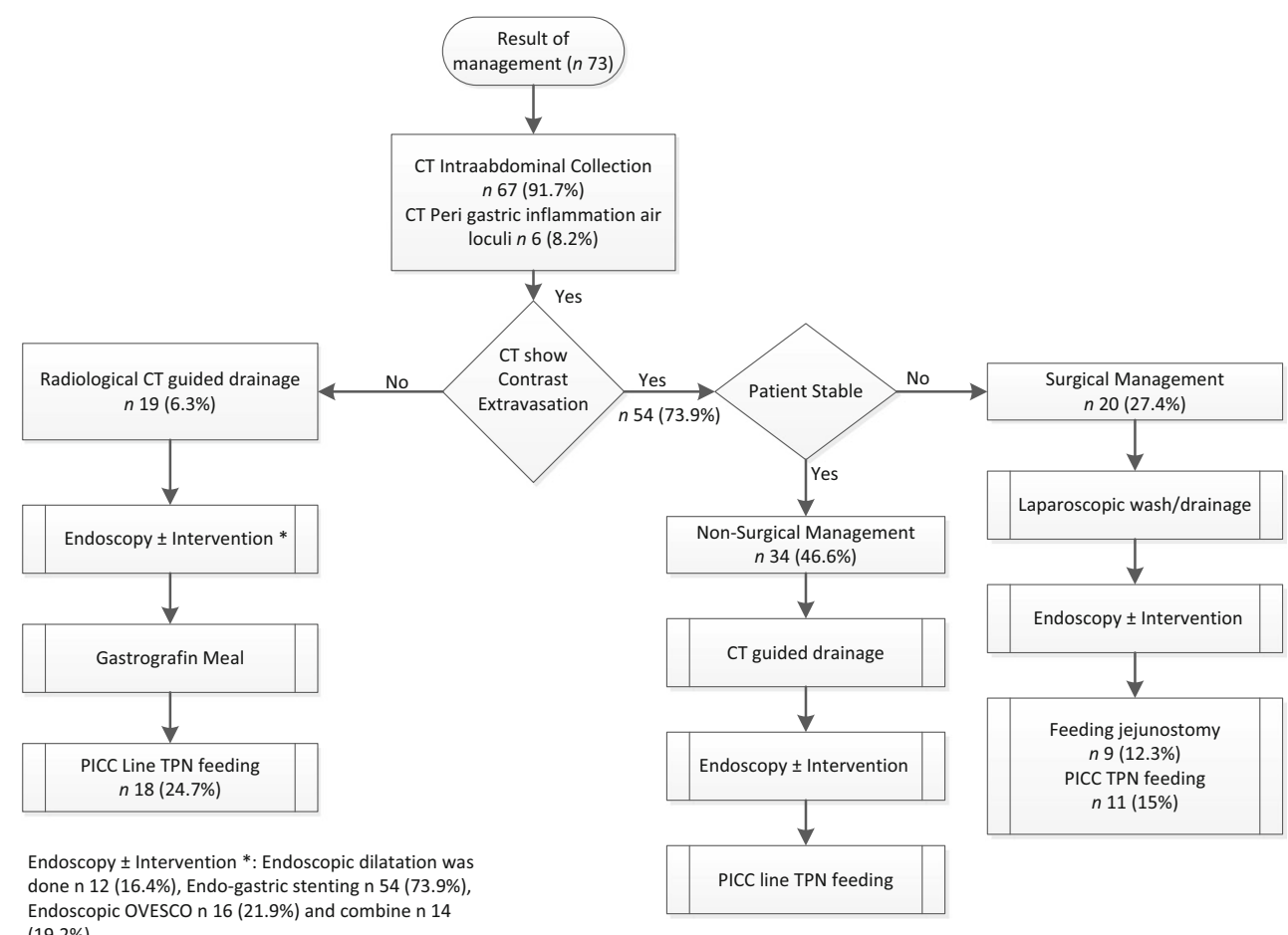

Endoscopic OVESCO n 16 (21.9\%) and combine $\mathrm{n} 14$ $(19.2 \%)$

An international debate exists regarding the use of endoscopic gastric stents. Patient's intolerance, cost, and potentially severe complications are the main concerns. Endoscopic stenting was done in $73.9 \%$ of patients (Fig. 2). All of them healed their leaks. Life-threatening stent complications occurred. Reporting of stent complication was out of the scope of this study.

\section{Predictors of Leak Resolution}

The management of leaks depletes various healthcare resources. Therefore, this study aimed to assess whether factors

Table 2 The outcomes and complications of post laparoscopic sleeve gastrectomy leak following our management algorithm in 68 patients

\begin{tabular}{ll}
\hline Outcome & $N(\%)$ \\
\hline Mortality & 0 \\
Resolution without conversion & $66(97.1)$ \\
Resolution after conversion & $2(2.9)$ \\
ICU & $22(30 \%)$ \\
Re-leak & $10(14.7)$ \\
Gastrocutaneous fistula & $2(2.9)$ \\
Deep venous thrombosis & $2(2.9)$ \\
PE & $1(1.5)$ \\
Splenic abscess & $2(2.9)$ \\
\hline
\end{tabular}

Results are based on number of patients who continued their treatment in our center (68 patients)

$N$, number of patients; $(\%)$, percentage; $I C U$, patients who required admission to the intensive care unit, $P E$, pulmonary embolism exist to decrease the time of resolution. In a large meta-analysis, the authors suggested that a higher BMI was associated with a higher risk of leak [9]. Al-Sabah and his colleagues also concluded that a high BMI and fever are predictors of mortality in patients with leak [11]. In our study, the exploration of potential prediction by patient's symptoms was out of the scope of this paper. However, analysis indicated that the baseline BMI had no effect on the time to resolution (HR 1,95\% CI 0.97-1.03) (Table 3).

In our practice, leak occurred in $0.4 \%$ after primary LSG and in $0.6 \%$ after revisional LSG. The risk of post LSG leak is higher in revisional surgeries due to improper tissue vascularity and diminished healing. Despite this concept, patients who underwent primary LSG, although statistically insignificant, had a similar time to resolution as those of revisional LSG (HR 1, 95\% CI 0.59-2.46) $P=0.64$. Interestingly, the number of fistulous openings also did not affect the resolution time; however, our clinical observation in patients with multiple fistulous openings demonstrated difficult and longer courses to resolution.

The fashioning of a feeding jejunostomy was a surgeon's decision. Otherwise, the patient was kept on TPN. Some colleagues avoided parenteral nutrition at any cost [7]. Statistical analysis indicated that patients on feeding jejunostomy had shorter time to resolution than those on TPN (Table 3). In our opinion, feeding through jejunostomy was superior to TPN in maintaining a good nutritional status and hence faster recovery, though comparing the complications and efficiencies between both methods was not studied in this paper.

The literature suggested the presence of sleeve stricture as a factor that affects the plan of management in leak patients [7]. 
Table 3 Predictive factors of leak healing post sleeve gastrectomy

\begin{tabular}{|c|c|c|c|c|c|c|}
\hline Factor & $\begin{array}{l}\text { Uni. analysis } \\
\text { (HR) }\end{array}$ & $P$ & $95 \% \mathrm{CI}$ & $\begin{array}{l}\text { Multi. analysis } \\
\text { (HR) }\end{array}$ & $P$ & $95 \% \mathrm{CI}$ \\
\hline Gender & 0.94 & 0.53 & $0.57-1.6$ & 0.90 & 0.73 & $0.53-1.52$ \\
\hline BMI & 1 & 0.71 & $0.98-1$ & 1 & 0.96 & $0.97-1.03$ \\
\hline Surg. days & 1 & 0.65 & $0.99-1.02$ & 1 & 0.54 & $0.99-1.02$ \\
\hline Procedure & 1.17 & 0.64 & $0.61-2.24$ & 1.21 & 0.59 & $0.59-2.46$ \\
\hline Fist.Opening & 0.53 & 0.23 & $0.22-1.25$ & 0.54 & 0.75 & $0.17-1.7$ \\
\hline $\begin{array}{l}\text { Surgical } \\
\text { management }\end{array}$ & 0.53 & 0.89 & $0.27-1.01$ & 1.1 & 0.89 & $0.64-1.88$ \\
\hline $\begin{array}{l}\text { Radiological } \\
\text { drainage }\end{array}$ & 0.7 & 0.17 & $0.18-0.42$ & 0.76 & 0.42 & $0.44-1.31$ \\
\hline $\begin{array}{r}\text { Endoscopic } \\
\text { dilatation }\end{array}$ & 0.5 & 0.047 & $0.25-0.99$ & 0.53 & 0.91 & $0.25-1.11$ \\
\hline TPN & 0.37 & 0.018 & $0.16-0.85$ & 0.34 & 0.026 & $0.13-0.88$ \\
\hline $\begin{array}{l}\text { Feeding } \\
\text { jejunostomy }\end{array}$ & 2.7 & 0.018 & $1.18-6.15$ & 2.4 & 0.038 & $1.049-5.54$ \\
\hline
\end{tabular}

All $P$ values and $95 \%$ CI for the hazard ratios are insignificant

Uni. analysis, univariate analysis; Multi. analysis, multi-variate analysis; (HR), hazard ratios; BMI, body mass index; Surg. days, days to presentation post surgery; procedure, primary vs revisional sleeve gastrectomy; Fist.Opening, number of fistulous opening in endoscopy; Surgical management, laparoscopic drainage and wash; Radiological drainage, radiological guided drainage; TPN total parenteral nutrition

$P$ values in itaics: significant value
In our group, twelve patients (16.4\%) had stricture. All underwent balloon dilatation combined with endo-gastric stent placement (Fig. 2). Those patients had 50\% increase in the resolution time compared with those who did not (HR = $0.5, P=0.047$ ) (Table 3). This finding might indicate stricture, increased the time to resolution despite endoscopic dilatation

\section{Limitations}

Despite the retrospective design in this manuscript, it represents the largest number of single-center experience in treating leak patients. To our knowledge, no paper has explored factors to decrease the time to leak resolution. Many possible essential factors related to the patients clinical and nutritional statuses as well as some techniques related factors could be of importance, but due to a risk of reduction of the statistical power, only eight factors have been studied. Complications of endoscopic stenting, feeding jejunostomy, and PICC line insertion combined with TPN were not included in this paper to narrow the focus of the manuscript. Further studies may present more detailed aspects of our experience in managing leak patients.

\section{Conclusion}

The management plan for post LSG leak is of multimodalities. Our clinical experience demonstrated less urge to perform extensive surgical interventions, at the cost of $8.8+0.72$ weeks to the resolution time. Patients on enteral feeding had a shorter time to heal their leaks, while patients with sleeve stricture had a longer time to resolution.

Acknowledgments Open access funding provided by the Qatar National Library. The authors would like to thank Professor Luigi Angrisani for his tremendous effort in the review and finalization of this paper and Mr. Arnel Alviz (systemanalyst, Hamad Medical CorporationQatar) who helped withthe data collection and statistical analysis. The publication of this article was funded by the Qatar National Library.

\section{Compliance with Ethical Standards}

Conflict of Interest The authors declare that they have no conflict of interest.

Ethical Approval Statement For this type of study, formal consent is not required.

Informed Consent Statement Does not apply.

Open Access This article is distributed under the terms of the Creative Commons Attribution 4.0 International License (http:// creativecommons.org/licenses/by/4.0/), which permits unrestricted use, distribution, and reproduction in any medium, provided you give appropriate credit to the original author(s) and the source, provide a link to the Creative Commons license, and indicate if changes were made.

\section{References}

1. Angrisani L, Santonicola A, Iovino P, et al. IFSO Worldwide Survey 2016: primary, endoluminal, and revisional procedures. Obes Surg. 2018;28(12):3783-94. 
2. Benedix F, Poranzke O, Adolf D, et al. Staple line leak after primary sleeve gastrectomy-risk factors and mid-term results: do patients still benefit from the weight loss procedure? Obes Surg. 2017;27(7):1780-8.

3. Brethauer SA, Hammel JP, Schauer PR. Systematic review of sleeve gastrectomy as staging and primary bariatric procedure. Surg Obes Relat Dis. 2009;5(4):469-75.

4. Abou RA, Basile M, El MH. Gastric leaks post sleeve gastrectomy: review of its prevention and management. World J Gastroenterol. 2014;20(38):13904-10.

5. Gagner M. Decreased incidence of leaks after sleeve gastrectomy and improved treatments. Surg Obes Relat Dis. 2014;10(4):611-2.

6. Deitel M, Crosby RD, Gagner M. The first international consensus summit for sleeve gastrectomy (SG). Obes Surg. 2008;18(5):48796.

7. Nimeri A, Ibrahim M, Maasher A, et al. Management algorithm for leaks following laparoscopic sleeve gastrectomy. Obes Surg. 2016;26(1):21-5.
8. Alizadeh RF, Li S, Inaba C, et al. Risk factors for gastrointestinal leak after bariatric surgery: MBASQIP analysis. J Am Coll Surg. 2018;227(1):135-41.

9. Parikh M, Issa R, McCrillis A, et al. Surgical strategies that may decrease leak after laparoscopic sleeve gastrectomy: a systematic review and meta-analysis of 9991 cases. Ann Surg. 2013;257:2317.

10. Csendes A, Braghetto I, León P, et al. Management of leaks after laparoscopic sleeve gastrectomy in patients with obesity. J Gastrointest Surg. 2010;14(9):1343-8.

11. Al-Sabah S, Ladouceur M, Christou N. Anastomotic leaks after bariatric surgery: it is the host response that matters. Surg Obes Relat Dis. 2008;4(2):152-7.

Publisher's Note Springer Nature remains neutral with regard to jurisdictional claims in published maps and institutional affiliations. 